\title{
Study of the pinned vortex fraction in the single crystal YBaCuO films
}

\author{
A. Hafid ${ }^{1}$, A. Taoufik ${ }^{1}$, A. Bouaaddi ${ }^{1}$, A. Tirbiyine ${ }^{1}$, M. Bghour ${ }^{1}$, B. Elmouden ${ }^{1}$, M. Boudhira ${ }^{1}$, \\ H. El hamidi ${ }^{1}$, H. Elouadi ${ }^{1}$, A. Nafidi ${ }^{1}$, H. Chaib ${ }^{2}$, E.H. Boudjema ${ }^{3}$ and M. Mahtali ${ }^{3}$ \\ ${ }^{1}$ Équipe des Matériaux Supraconducteur à Haute Tc, Université IBN ZOHR, Faculté des Sciences d'Agadir, \\ Maroc \\ ${ }^{2}$ Faculté polydisciplinaire, Université Ibn Zohr, BP. 83, 45002 Ouarzazate, Maroc \\ ${ }^{3}$ Laboratoire Couches mince et interfaces, Département de physique, Faculté des sciences Exactes, Université \\ Monteri- Constantine, Campus Chaab-Erassas, 25000 Constantine, Algérie
}

\begin{abstract}
In this work we intend to study the pinned vortex fraction in type II superconductors. The sample used is the $\mathrm{YBa} 2 \mathrm{Cu} 3 \mathrm{O} 7-\gamma$. In continuation one will study the variations of this fraction according to the transport current density for two values of magnetic field $(\mathrm{H}=0.6 \mathrm{~T}$ and $\mathrm{H}=1.2 \mathrm{~T})$, and different values of temperature. The studied sample is a monocristalline $\mathrm{YBaCuO}$ thin film deposited by the ablation laser method on the surface (001) of a SrTiO3 substrate. In zero magnetic field, the resistance vanished a $\mathrm{Tc}=90 \mathrm{~K}$. The $\mathrm{C}$-axis of $\mathrm{YBaCuO}$ is perpendicular to surface of the film. Electrodes of measurement are in gold and deposited on the surface of the sample in situ by evaporation. The film has a thickness of $400 \mathrm{~nm}$, and a width $7.53 \mu \mathrm{m}$. The distance between electrodes of power measurement is $135 \mu \mathrm{m}$. Contact resistances were less than $1 \Omega$.
\end{abstract}

\section{INTRODUCTION}

The phenomena of flux motion and flux flow resistivity are really the heart of the dissipative mechanism in superconductors.

The transport current interacts with the flux quanta to exert a Lorentz force on each flux vortex of the form

$$
\mathrm{f}_{1}=\mathrm{j} * \Phi_{0} .
$$

In the above expression $f_{1}$ is the force per unit length on the flux vortex, $J_{t}$ is the transport current density and $\Phi_{0}$ is the quantum of flux in the vortex. The moving flux vortices will induce an electric field E, given by [1]:

$$
\mathrm{E}=\mathrm{V}_{1}(1-\mathrm{p})=\mathrm{V}_{1} * n * \Phi_{0}(1-\mathrm{p}) .
$$

Where $\mathrm{V}_{l}$, is the velocity of flux lines.

$\mathrm{n}$ is the number of flux lines per unit area such that $\mathrm{B}=\mathrm{n} \Phi_{0}$.

p: Pinned vortex fraction.

Since there is no pinning force to balance the Lorentz force, we might assume that the flux lines accelerate and thus E would increase with time. However, the observed voltage indicates that $\mathrm{E}$ is constant in time implying a drag force must exist to balance the Lorentz force. This force balance was proposed by Kim in the form [2-5]

$$
\eta \cdot \mathrm{V}_{1}=\mathrm{f}_{1}=\mathrm{J} * \Phi_{0}
$$

$\eta$ is a viscous damping constant.

Equations (2) and (3) can be combined to define the "flux flow resistivity":

$$
\rho_{\mathrm{f}}=\frac{\mathrm{E}}{\mathrm{J}}=\mathrm{B} \Phi_{0} \frac{(1-\mathrm{p})}{\eta} .
$$

The critical state then is just the state where the Lorentz force is exactly equal to the pinning force and is governed by the equation:

$$
J_{\mathrm{c}} \cdot \Phi_{0}=f_{\mathrm{p}} .
$$

The induced electric field is:

$$
\mathrm{E}=\mathrm{B} \Phi_{0} \frac{(1-\mathrm{p}) \mathrm{J}}{\eta} .
$$

So far, no mention has been made of exactly what is the dissipative mechanism due to a moving vortex. Experimental measurements of $\rho_{f}$ by Kim, Hempstead and Strnad show that it is a function of temperature; it is well correlated by the expression [2].

$$
\rho_{\mathrm{f}}=\rho_{\mathrm{n}} \cdot \frac{\mathrm{H}}{\mathrm{H}_{\mathrm{c} 2}} .
$$

Where $\rho_{\mathrm{n}}$ is the resistivity of the normal material. The ratio $\mathrm{H} / \mathrm{H}_{\mathrm{c} 2}$ is just the fraction of the normal core area. This is a very interesting result because it implies that the dissipation is due to the transport current flowing through the normal cores of the moving flux vortices

$$
\mathrm{E}=\rho_{f} \cdot \mathbf{J}=\left(\rho_{\mathrm{n}} \cdot \frac{\mathrm{H}}{\mathrm{H}_{\mathrm{c} 2}}\right) \mathbf{J}(1-\mathrm{p}) .
$$

\section{RESULTS AND DISCUSSION}

\subsection{Determination of the pinned vortex fraction $p$}

The Pinned flux fraction of vortex $\mathrm{p}$ is determined from experimental data characteristics $\mathrm{E}(\mathrm{J})$ and using the expression (8) where it is based on F. Irie, and $\mathrm{K}$. Yamafuji [6]:

$$
\rho_{\mathrm{n}}=3 \mu \Omega \cdot \mathrm{cm}
$$

And

$$
\mu_{0} \mathrm{H}_{\mathrm{c} 2}=\frac{\Phi_{0}}{2 \pi \xi^{2}}=14,7 \mathrm{~T}
$$




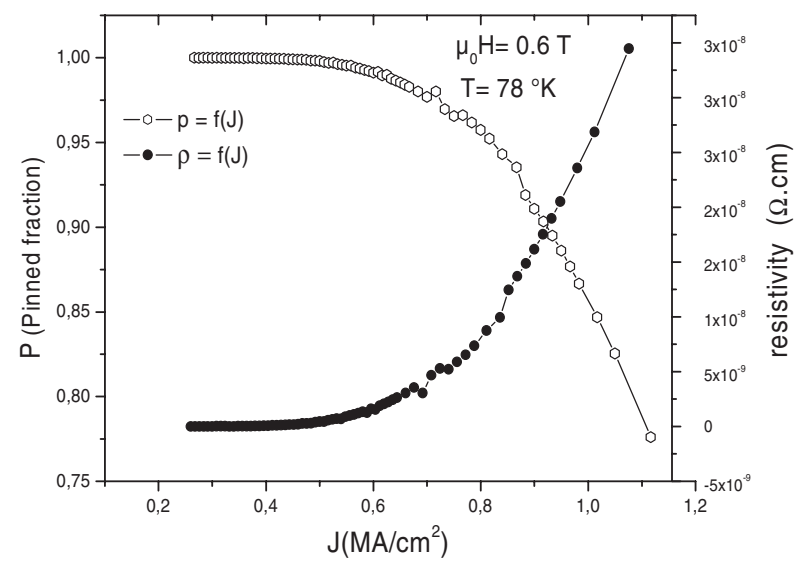

Figure 1. The resistivity $\rho$ and the pinned flux fraction of vortex $\mathrm{p}$ versus transport current density $\mathrm{J} \mu 0 \mathrm{H} 0.6 \mathrm{~T} / /(\mathrm{ab})$ plan and $T=78 \mathrm{~K}$.

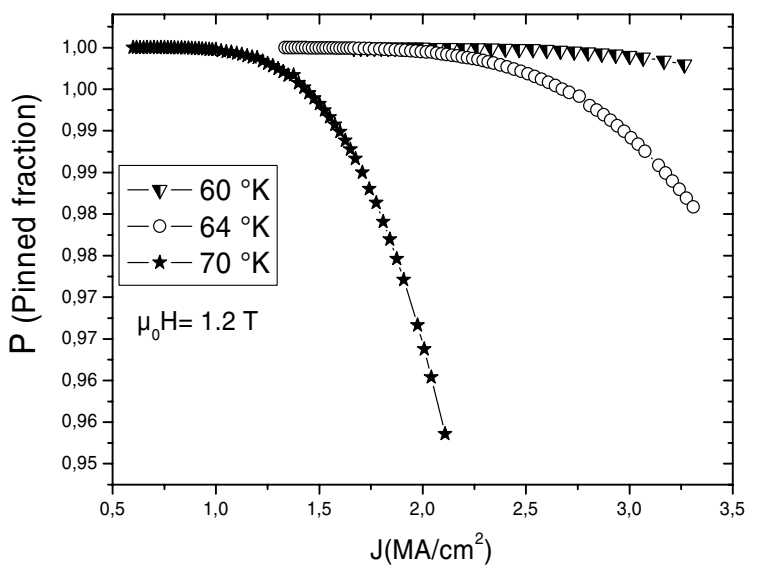

Figure 2. Pinned vortex fraction versus $\mathrm{J}$ for different values of the temperature in a magnetic field of $1.2 \mathrm{~T}$ parallel to the planes $a b$.

Figure (1) shows the variations of the resistivity $\rho$ and the Pinned flux fraction of vortex $p$ versus transport current density $\mathrm{J}$ we obtained for a magnetic field $0.6 \mathrm{~T}$ at a temperature of $78 \mathrm{~K}$.

The curve giving the variation of the resistivity $\rho(\mathrm{J})$ shows two regimes [7]:

Flux creep regime for low electric current densities. J

Flux flow regime for high current densities where changes in $\rho(\mathbf{J})$ are almost linear

These two regimes are also appearing in variation in the fraction of pinned vortex $\mathrm{p}(\mathrm{J})$. For low current densities the vortices are almost anchored by defects in the sample giving a value to the pinned vortex fraction very near to 1 , for high values of $\mathrm{J}$, the system enters the regime of flux flow and the pinned vortex fraction decreases.

\subsection{Variation of $\mathbf{p}$ as a function of temperature and magnetic field}

\subsubsection{Influence of temperature}

Figure (2) shows the variations of the pinned vortex fraction according to $\mathrm{J}$ for three values of temperature

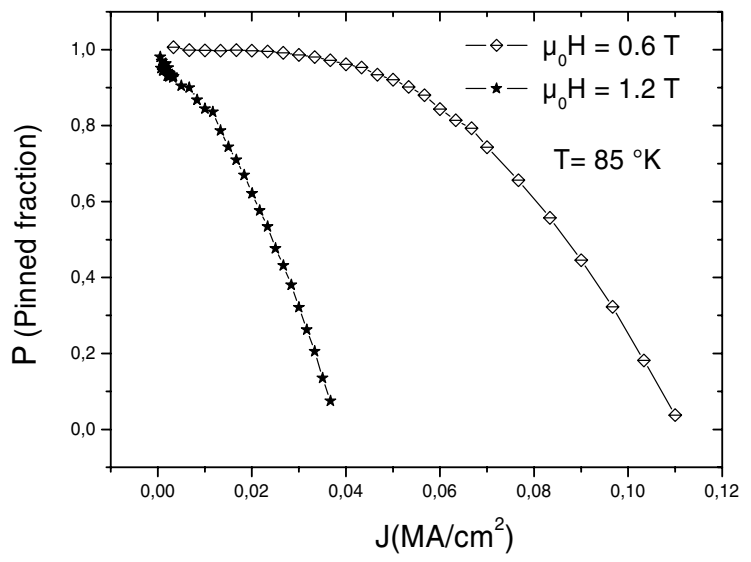

Figure 3. Variation of the pinned flux fraction for two values of the magnetic field 0.6 and $1.2 \mathrm{~T}$ at $\mathrm{T}=85^{\circ} \mathrm{K}$.

$(60 \mathrm{~K}, 64 \mathrm{~K}$ and $70 \mathrm{~K})$ in a magnetic field of $1.2 \mathrm{~T}$ parallel to the ab planes.

\subsubsection{Influence of the magnetic field}

Figure (3) shows the variations of the pinned flux fraction according to $\mathrm{J}$ for two values of the magnetic field $0.6 \mathrm{~T}$ and 1,2 T parallel to the planes ab and a temperature of $85 \mathrm{~K}$.

When the applied magnetic field increases, for a fixed temperature, the pinned vortex fraction decreases. The vortex can spend based on the magnetic field of the state completely pinned in the state where they are completely free to move.

\section{CONCLUSION}

The random jumping of flux vortices from one site to another causes the flux creep due to vibration caused by the thermally induced vibrations. It manifests itself by slow decay of a trapped magnetic field and/or by a measurable resistive voltage. This phenomenon has been described by the Anderson-Kim [8,9] flux creep theory which assumes the vortices move in bundles. However this effect can lead to a potentially more dramatic effect called a flux jump.

Our results show that the pinned fraction decreases with increasing temperature for a fixed magnetic field. This justifies the fact that the thermal activation of vortices depinned their pinning centers. When the applied magnetic field increases, for a fixed temperature, the pinned vortex fraction decreases. The vortex can spend based on the magnetic field of the state completely pinned in the state where they are completely free to move.

\section{References}

[1] G. J. Van Gurp Physical Review 166 (1968) 436-446.

[2] Kim, Y.B. Hempstead, C.F., and Strnar, A.R., Physical Review, Vol. 131,2486(1963). 
[3] Friedel J, De Gennes PG, Matricon J. Nature of the driving force in flux creep phenomena. Appl Phys Lett; 2: 119-21 (1963).

[4] Silcox J, Rolins RW. Hysteresis in hard superconductors. Appl Phys Lett; 2: 231-3 (1963).

[5] Gorter CJ. On the partial persistence of superconductivity at very high magnetic fields and current densities. Phys Lett; 2, 26-7 (1962).
[6] Irie, F., and Yamafuji, K., Journal of the Physical Society of Japan, vol. 23, $\mathrm{N}^{\circ} 2,255$ (19).

[7] GMELIN (F.) Thermal properties of HTSC. Vol.2.p.95, Studies of HTSC, NovaSciences Publ. (1989).

[8] P. W. Anderson, Phys. Rev. Lett. 9, 309 (1962).

[9] P. W. Anderson, Y. B. Kim, Rev. Mod. Phys. 36, 39 (1964). 